\title{
Constrained peptides mimic a viral suppressor of RNA silencing
}

Arne Kuepper ${ }^{1,2}$, Niall M. McLoughlin ${ }^{3}$, Saskia Neubacher ${ }^{3}$, Estel Collado-Camps ${ }^{4}$, Chandran Nithin ${ }^{5}$, Sunandan Mukherjee ${ }^{5}$, Lucas Bethge ${ }^{6}$, Janusz M. Bujnicki ${ }^{5}$, Roland Brock ${ }^{4}$, Stefan Heinrichs ${ }^{7}$, Tom N. Grossmann ${ }^{1,2,3^{*}}$

${ }^{1}$ Chemical Genomics Centre of the Max Planck Society, Dortmund, 44227, Germany,

${ }^{2}$ Department of Chemistry and Chemical Biology, Technical University Dortmund, Dortmund, 44227, Germany,

${ }^{3}$ Department of Chemistry and Pharmaceutical Sciences, Amsterdam Institute of Molecular and Life Sciences (AIMMS), Vrije Universiteit Amsterdam, Amsterdam, $1081 \mathrm{HZ}$, The Netherlands,

${ }^{4}$ Department of Biochemistry, Radboud Institute for Molecular Life Sciences, Radboud University, Nijmegen Medical Center, Nijmegen, 6525 GA, The Netherlands,

${ }^{5}$ Laboratory of Bioinformatics and Protein Engineering, International Institute of Molecular and Cell

Biology in Warsaw, Warsaw, 02-109, Poland,

${ }^{6}$ Silence Therapeutics GmbH, Robert-Rössle-Str. 10, 13125 Berlin, Germany,

${ }^{7}$ University Hospital Essen, Institute for Transfusion Medicine, Essen, 45147, Germany.

*To whom correspondence should be addressed. Email: t.n.grossmann@vu.nl

\section{ABSTRACT}

The design of high-affinity, RNA-binding ligands has proven very challenging. This is due to the unique structural properties of RNA, often involving relatively polar surfaces and structural dynamics. In addition, the frequent lack of well-defined binding pockets complicates the development of small molecule binders. This has triggered the search for alternative scaffolds of intermediate size. Among these, peptide-derived molecules represent appealing entities as they can mimic structural features also present in RNA-binding proteins. However, the application of peptidic RNA-targeting ligands is hampered by a lack of design principles and their inherently low bio-stability. Here, the structurebased design of constrained $\alpha$-helical peptides derived from the viral suppressor of RNA silencing $T A V 2 b$ is described. We observe that the introduction of two inter-side chain crosslinks provides peptides with increased $\alpha$-helicity and protease stability. One of these modified peptides (B3) shows high affinity for different double-stranded RNA structures including a palindromic siRNA as well as microRNA-21 and its precursor pre-miR-21. Notably, B3 binding to pre-miR-21 inhibits Dicer processing in a biochemical assay. As a further characteristic this peptide also exhibits cellular entry. Our findings show that constrained peptides can efficiently mimic RNA-binding proteins rendering them potentially useful for the design of bioactive RNA-targeting ligands. 


\section{INTRODUCTION}

Viruses represent a universal infectious threat to all forms of life, from bacteria to fungi, plants and animals. In response, these organisms have evolved numerous mechanisms to fend off viral attack (1). One such defense mechanism in eukaryotes is RNA silencing, a central cellular process that triggers the degradation of viral double-stranded RNA (ds RNA) arising from replication intermediates or self-complementary viral genome sequences $(2,3)$. In this process, Dicer-like proteins cleave long ds RNA into short fragments ( $19-23$ base pairs long) known as small interfering RNAs (siRNAs). One of the two siRNA strands can then be incorporated into the RNA-induced silencing complex (RISC) which recognizes and cleaves complement viral messenger RNA (mRNA) (4). To suppress RNA silencing, viruses have evolved proteins which directly bind to siRNA structures thereby preventing RISC incorporation (5). Such viral suppressors of RNA silencing are diverse in structure and size, and belong to the large group of RNA-binding proteins (6-8). This group of proteins features a broad spectrum of functionalities beyond post-transcriptional regulation, and several efforts have been made to harness their scaffolds to design biomolecular tools that recognize specific RNA structures and sequences (9-12).

In contrast to the progress made in re-engineering RNA-binding proteins, the design of small molecular scaffolds that recognize RNA structures has proven more challenging (13-16). This is due to the unique structural features of RNA often involving dynamic and polar surfaces which tend to lack well-defined binding pockets. For this reason, alternative scaffolds of intermediate size have been exploited (17-19). Among those, peptide-derived molecules represent appealing entities as they can mimic structural features found in RNA-binding proteins. To date, peptide-based approaches have seen limited RNA-targeting applications. Examples have used the HIV-1 RNA-binding peptides, Tat and Rev, as starting points to apply different strategies to stabilize their bioactive conformation including lactam-bridge formation, head-to-tail cyclization, and thioether crosslinking (20-24). Moreover, the screening of modified peptide libraries has resulted in the discovery of novel RNAbinding ligands (25-27). However, the cellular uptake of such peptide-derived ligands has been a limiting factor.

For the development of inhibitors of protein-protein interactions, peptide-derived ligands have been widely used (28). In those cases, protein fragments have been stabilized in their bioactive conformation to provide medium-sized, cell-permeable ligands (28-30). Among the most common design approaches is the stabilization of $\alpha$-helical peptide conformations via inter-side chain crosslinks (30-32). In particular, the design of so-called hydrocarbon-stapled peptides has seen broad application $(32,33)$. In this setup, macrocyclization is conducted between a pair of $\alpha$-methylated, nonnatural amino acids featuring terminal alkene side chains. Through ring-closing metathesis, these alkenes are connected to form an intramolecular crosslink (staple) which supports the helical fold (34, 35). So far, hydrocarbon-stapled peptides have not been used to target RNA and it is unclear if such structures are suitable for RNA-targeting. Also, questions remain over the general applicability of peptidomimetics as RNA-binding ligands.

Herein, we describe the design of hydrocarbon-stapled peptides derived from the tomato aspermy virus $2 \mathrm{~b}$ (TAV2b) protein, a viral suppressor of RNA silencing (36). Using a reported crystal 
structure of TAV2b bound to ds siRNA (37), we identified a mainly $\alpha$-helical 33-mer peptide that binds ds RNA with moderate affinity. To constrain the $\alpha$-helical conformation, we developed a series of mono- and double-stapled peptides among which we identified a protease-stable and cell-permeable double-stapled analog with high affinity for a palidromic siRNA. Notably, we also observe binding of this stapled peptide to the microRNA-21 duplex (miR-21) and its precursor, pre-miR-21, which both share structural features with siRNA. In biochemical assays, we observe that pre-miR-21 binding inhibits Dicer-mediated maturation.

\section{MATERIAL AND METHODS}

\section{Abbreviations}

Fmoc: fluorenylmethyloxycarbonyl, SPPS: solid-phase peptide synthesis, EMSA: electrophoretic mobility shift assay; EDTA: ethylenediaminetetraacetic acid; PBS: phosphate buffered saline (137 mM $\mathrm{NaCl}, 2.7 \mathrm{mM} \mathrm{KCl}, 10 \mathrm{mM} \mathrm{Na}_{2} \mathrm{HPO}_{4}, 2 \mathrm{mM} \mathrm{KH}_{2} \mathrm{PO}_{4}$, pH 7.4); TFA: trifluoroacetic acid; ACN: acetonitrile; DMSO: dimethyl sulfoxide; HEPES: 4-(2-hydroxyethyl)-1-piperazineethanesulfonic acid; FCS: fetal calf serum; qRT-PCR: quantitative reverse transcription polymerase chain reaction; PAGE: polyacrylamide gel electrophoresis.

\section{Oligonucleotides}

The sequences and names of all oligonucleotides used in this study are presented in Supplementary Table S1. High-performance liquid chromatography (HPLC)-purified oligonucleotides were purchased from Eurofins Genomics and NOXXON Pharma. Transfer-RNA (t-RNA) from baker's yeast was purchased from Hoffmann La Roche. For quantification, the ultraviolet (UV) absorbance of the oligonucleotides was measured in the buffer of the corresponding experiment using a V-550 ultraviolet/visible (UV/Vis) spectrophotometer (Jasco). Respective concentrations were calculated with a molar extinction coefficient at $\lambda=260 \mathrm{~nm}$, determined according to the nearest-neighbor model using published parameters for oligonucleotides (38-42). RNA duplexes were heated to $95^{\circ} \mathrm{C}$ for 10 min and slowly cooled to room temperature (RT) for $1 \mathrm{~h}$ prior to experiments.

\section{Solid-phase peptide synthesis}

Peptides were synthesized according to standard Fmoc-chemistry for SPPS (43) using HCTU (O-(6chlorobenzotriazol-1-yl)-N,N,N',N'-tetramethyluronium hexafluorophosphate), PyBOP (benzotriazol-1yl-oxy-tris-pyrrolidino-phosphonium hexafluorophosphate), COMU (1-cyano-2-ethoxy-2oxoethylidenaminooxy)dimethylamino-morpholino-carbenium hexafluorophosphate) and Oxyma (ethyl cyano(hydroxyimino)acetate) as coupling reagents. For more detailed information about peptide synthesis and characterization see Supplementary Methods, Supplementary Table S2 and Supplementary Figures S22-S26.

\section{Electrophoretic mobility shift assay}

Electrophoretic mobility shift assays (EMSAs) were performed using a Bio-Rad Mini-Protean gel system paired with a direct current (DC) power source (PowerPac ${ }^{\mathrm{TM}} \mathrm{HC}$, Bio-Rad). Typically, $6 \mu \mathrm{L}$ solutions containing ds RNA $(c=3 \mu \mathrm{M})$ and $N$-terminally fluorescein-isothiocyanate-labeled peptide $(c$ 
$=6 \mu \mathrm{M})$ were incubated for 1 hour at $4{ }^{\circ} \mathrm{C}$ in a binding buffer of $10 \mathrm{mM}$ Tris $(\mathrm{pH}=8.0), 0.5 \mathrm{mM}$ EDTA, $12.5 \%$ glycerol, and $150 \mathrm{mM} \mathrm{KCl}$. After incubation, bound RNA was resolved from peptide-free RNA using $10 \%$ non-denaturing polyacrylamide gels at $120 \mathrm{~V}$ in running buffer $(25 \mathrm{mM}$ Tris $(\mathrm{pH}=8.0), 200$ $\mathrm{mM}$ glycine, $20 \mathrm{mM} \mathrm{NaCl}$ ) at $4{ }^{\circ} \mathrm{C}$ for 1 hour. Visualization of fluorescent gel-bound species was conducted using a Typhoon Trio + fluorescence scanner (GE Healthcare Life Sciences). For nucleic acid visualization gels were stained using $2 \mu \mathrm{L}$ of SYBR ${ }^{\mathrm{TM}}$ gold nucleic acid gel dye (Molecular Probes) in $20 \mathrm{~mL}$ of $1 \times$ TAE buffer ( $40 \mathrm{mM}$ Tris, $1 \mathrm{mM}$ EDTA, $20 \mathrm{mM}$ acetic acid) for 45 minutes at RT before being visualized using a FluorChem ${ }^{\mathrm{TM}}$ gel documentation system (Alpha Innotech).

\section{Isothermal titration calorimetry}

Isothermal titration calorimetry (ITC) was conducted using a MicroCal VP-ITC (Malvern Panalytical). Before measurement, oligonucleotide and peptide samples were dissolved in 1xPBS and degassed using a ThermoVac (Malvern Panalytical) and heated to $30^{\circ} \mathrm{C}$. $N$-terminally acetylated peptides $(C=3$ $\mu \mathrm{M}-12 \mu \mathrm{M})$ were then transferred into the sample cell and oligonucleotides $(c=12 \mu \mathrm{M}-72 \mu \mathrm{M})$ were transferred into the syringe. 35 injections per measurement were performed at $30^{\circ} \mathrm{C}(8 \mu \mathrm{L}$ injection volume, $2 \mathrm{~s}$ injection time, $180 \mathrm{~s}$ spacing, high feedback mode) with an initial delay of $60 \mathrm{~s}$ and a stirring speed of $307 \mathrm{rpm}$. Measurements were performed in triplicate. Using the MicroCal LLC ITC software (Origin; OriginLab Corporation), the heat associated with each injection was calculated by integrating the area under the curve in microcalories per second $(\mu \mathrm{ca} / \mathrm{s})$ versus time $(\mathrm{min})$ for each injection and then normalized to concentration. A 'single set of identical binding sites' model was used to fit the binding curves from which thermodynamic parameters $(\Delta G, \Delta H, \Delta S)$ and binding parameters $\left(N\right.$ and $\left.K_{d}\right)$ were obtained.

\section{Circular dichroism spectroscopy \& $T_{\mathrm{m}}$ determination}

Circular dichroism (CD) spectra of $N$-terminally acetylated wt33 and ds RNA were recorded with a Jasco J-1500 spectropolarimeter (Jasco) equipped with a programmable Peltier thermostat in a stoppered quartz cuvette $(10 \mathrm{~mm}$; Hellma). Samples of pal-RNA $(c=2 \mu \mathrm{M})$ and wt33 $(c=4 \mu \mathrm{M})$ were prepared in a buffer of $10 \mathrm{mM}$ sodium phosphate buffer and $100 \mathrm{mM} \mathrm{NaCl}(\mathrm{pH}$ 7.4). For each sample $10 \mathrm{CD}$ spectra were measured between $\lambda=200 \mathrm{~nm}$ and $\lambda=350 \mathrm{~nm}$ with continuous scan mode (1 mdeg sensitivity, $1.0 \mathrm{~nm}$ resolution, $1.0 \mathrm{~nm}$ bandwidth, $2 \mathrm{~s}$ integration time, $100 \mathrm{~nm} / \mathrm{min}$ scan rate). Obtained spectra were averaged and then subtracted from a reference buffer spectrum. CD data were normalized to oligonucleotide strand concentration using Formula 1:

$$
\varepsilon l-\varepsilon r=\Delta \varepsilon=\frac{\theta}{32980 \cdot c \cdot l},
$$

where $\theta=$ observed ellipticity $/$ mdeg, $c=$ DNA strand concentration $/ \mathrm{mol} \cdot \mathrm{L}^{-1}$ and $I=$ path length $/ \mathrm{cm}$.

Melting temperature $\left(T_{\mathrm{m}}\right)$ determination was conducted using the same instrumentation and sample preparation, where ellipticity $(\theta=267 \mathrm{~nm})$ was measured by ramping the temperature from $15-90^{\circ} \mathrm{C}$ $\left(4^{\circ} \mathrm{C} /\right.$ minute ramp, $\pm 0.05^{\circ} \mathrm{C}$ equilibration tolerance, 6 seconds delay after equilibration). Points were taken every $0.5^{\circ} \mathrm{C}$. Raw data were normalized as described above and $T_{\mathrm{m}}$-values were determined using the CDpal program (44) before being plotted in Prism 5.0 (GraphPad). 
Spectra of $\mathrm{N}$-terminal fluorescein-isothiocyanate-labeled peptides were recorded with a J-715 circular dichroism spectrometer (Jasco) using a stoppered quartz cuvette (1 mm; Hellma). Peptide samples $(c=75 \mu \mathrm{M})$ were prepared in $10 \mathrm{mM}$ disodium hydrogen phosphate buffer $(\mathrm{pH}=7.4)$. For each sample $10 \mathrm{CD}$ spectra were measured between $\lambda=190 \mathrm{~nm}$ and $\lambda=260 \mathrm{~nm}$ with continuous scan mode (10 mdeg sensitivity, $1.0 \mathrm{~nm}$ resolution, $1.0 \mathrm{~nm}$ bandwidth, $2 \mathrm{~s}$ integration time, $50 \mathrm{~nm} / \mathrm{min}$ scan rate). Obtained spectra were averaged, then subtracted from a reference buffer spectrum and smoothed using a fast Fourier transform filter (FFT filter). The mean residue ellipticity $[\Theta] / \mathrm{deg} \cdot \mathrm{cm}^{2} \cdot \mathrm{dmol}^{-1} \cdot 10^{4}$ was calculated using Formula 2 (below) and plotted against wavelength $\lambda / \mathrm{nm}$. The ratio of secondary structure elements was calculated with CDNN using the PEPFIT set of reference spectra for secondary structure determination $(45,46)$.

$$
[\Theta]_{m}=\frac{\Theta}{c \cdot d \cdot 10000}
$$

Given that: $\frac{c_{g}}{M R W}$, with $M R W=\frac{M}{N}$,

where $[\Theta]_{\mathrm{m}}=$ mean molar ellipticity per peptide bond $/$ degree $\cdot \mathrm{cm}^{2} \cdot \mathrm{dmol}^{-1} \cdot 10^{4}, \Theta=$ measured ellipticity / degree, $c$ = peptide concentration $/ \mathrm{mol} \cdot \mathrm{L}^{-1}, d=$ optical path length $/ \mathrm{cm}, c_{g}=$ mass concentration / $\mathrm{g} \cdot \mathrm{L}^{-1}, M R W=$ mean residue weight, $M=$ molar mass of the peptide $/ \mathrm{g} \cdot \mathrm{mol}^{-1}$, and $N=$ number of peptide bonds.

\section{Protease stability assay}

Protease stability assays were performed similarly to a previously published protocol (47). Stocks of $\mathrm{N}$-terminally fluorescein-isothiocyanate-labeled peptides were prepared to a concentration of $55.5 \mu \mathrm{M}$ in assay buffer ( $10 \mathrm{mM}$ Tris, $\mathrm{pH}=8.0,150 \mathrm{mM} \mathrm{NaCl}$ ) and separately stocks of proteinase $\mathrm{K}$ also in assay buffer $\left(c=100 \mu \mathrm{g} \cdot \mathrm{mL}^{-1}\right)$. To begin each experiment, peptide and proteinase $\mathrm{K}$ stocks were mixed (final concentrations: $c=10 \mu \mathrm{g} \cdot \mathrm{mL}^{-1}$ proteinase $\mathrm{K}, c=50 \mu \mathrm{M}$ fluorescein-isothiocyanatemodified peptide; final volume $=500 \mu \mathrm{L}$ ) and incubated at room temperature. At each time point, 50 $\mu \mathrm{L}$ of the reaction mixture was quenched by adding $100 \mu \mathrm{L}$ of TFA/ACN/H $\mathrm{H}_{2} \mathrm{O}(\mathrm{v} / \mathrm{v} / \mathrm{v}, 1 / 49 / 49)$ and subsequently incubated for 2 minutes on ice. Following centrifugation ( 15 minutes, $13200 \mathrm{rpm}, 4{ }^{\circ} \mathrm{C}$ ) samples $(45 \mu \mathrm{L})$ were monitored by analytical HPLC using a linear gradient of $10-60 \%$ HPLC solvent $\mathrm{B}$ over 20 minutes. The amount of remaining peptide was quantified via absorbance at $\lambda=$ $210 \mathrm{~nm}$ (intact peptide / \%) and plotted against time $(t / \mathrm{h})$. Half-life $\left(t_{1 / 2}\right)$ was calculated by fitting the data to an exponential decay using the software Prism 5.0 (GraphPad).

\section{Peptide uptake}

$2 \times 10^{6} \mathrm{~K} 562$ cells were plated per well in 48 -well plates in presence of $\mathrm{N}$-terminally fluoresceinisothiocyanate-labeled peptides ( $c=1 \mu \mathrm{M}, 0.5 \% \mathrm{DMSO}$ ) in a total volume of $300 \mu \mathrm{L}$. After 90 minutes, cells were treated with proteinase $\mathrm{K}\left(2 \mu \mathrm{g} \cdot \mathrm{mL}^{-1}\right)$ for 10 minutes to remove non-incorporated peptides. Cells were extensively washed and total uptake of labeled peptide was measured by flow cytometry (Cytoflex, Beckman Coulter with the CytExpert software version 2.3). For each peptide 20,000 events / sample were collected with $>85 \%$ viable cells (forward/side scatter) that were considered for 
analysis using a 525/40 filter. DMSO was used as a control to distinguish general DMSO-related and peptide-specific effects. Each experiment was performed in triplicate.

\section{Pull-down}

$2 \times 10^{6} \mathrm{~K} 562$ cells were incubated with $N$-terminally biotinylated peptides $(c$ (peptide) $=10 \mu \mathrm{M}, t=2.5$ hours) for uptake on a rotating wheel at $37^{\circ} \mathrm{C}$. The cells were extensively washed in 1 XPBS and lysed in lysis buffer (25 mM HEPES, pH = 7.5, $150 \mathrm{mM} \mathrm{NaCl}, 0.05 \%$ IGEPAL CA-630, $1 \mathrm{mM}$ EDTA) supplemented with $0.5 \mathrm{U} / \mu \mathrm{L}$ RNAse inhibitor (Superase-in, Thermofisher). The lysate was cleared by centrifugation at $21,000 \times \mathrm{g}\left(4^{\circ} \mathrm{C}\right)$, an aliquot of the supernatant was removed and re-suspended in Trizol, while the remaining was incubated with streptavidin-coupled magnetic beads (Dynabeads M280, ThermoFisher) on a rotating wheel $\left(2 \mathrm{~h}, 4^{\circ} \mathrm{C}\right)$. After magnetic separation and washing $(3 \mathrm{x}$ in lysis buffer), the beads were re-suspended in $20 \mu \mathrm{L}$ lysis buffer, re-suspended in Trizol

(ThermoFisher), and $400 \mathrm{ng}$ transfer-RNA was added per sample as a carrier. RNA was extracted from Trizol using the standard protocol and the qRT-PCR was performed according to a previously published protocol (11) using the primers h-miR21-3p-FW (CAGCAACACCAGTCGATG), h-miR213p-RV (GGTCCAGTTTTTTTTTTTTTTTACAG), h-miR21-5p-FW (GCAGTAGCTTATCAGACTGATG), h-miR21-5p-RV (GGTCCAGTTTTTTTTTTTTTTTTCAAC) in a CFX96 real time machine (Bio-Rad). The relative enrichment was calculated as a ratio of copies in the immunoprecipitate over input normalized to the enrichment of the control peptide D1.

\section{Modeling of wt33 peptide and RNAs}

Structural models of miR-21 and pre-miR-21 RNAs were generated by a stepwise modeling approach (48). Briefly, the initial model was generated with the ModeRNA server (49) using the loop region from the nuclear magnetic resonance (NMR) structure (PDB ID: 5UZT) (50) as the template. It was then refolded with SimRNA (51), a method for simulations of RNA folding which uses a coarse-grained representation and relies on the Monte Carlo method for sampling the conformational space. SimRNA employs a statistical potential to approximate the energy using additional restraints on the secondary structure taken from miRBase database (52). Finally, the best-scoring model from SimRNA was optimized in the full-atom representation with QRNAS (53). The starting model of the wt33 peptide was generated based on the crystal structure of the TAV2b/siRNA complex (PDB ID: 2ZI0) (37). Models of the peptide/RNA complexes were generated using SimRNP, an extension of SimRNA (51), which uses a representation of protein structures as in REFINER (54) and an energy function derived from experimentally solved RNA and RNP structures. Both miR-21 and pre-miR-21 RNAs were simulated in the presence of single, double, and triple copies of the wt33 peptide.

For best-scored complexes generated from SimRNP simulations all-atom molecular dynamics (MD) simulations were carried out for miR-21 and pre-miR-21 RNAs with complexes of one, two, and three copies of wt33 peptide using the Amber 18 program suite (55). The starting structures for the simulation were prepared using tleap in a truncated octahedral box of $10 \AA$ allowance along with TIP3P water model (56). A combination of two force-fields, Amber ff14sb force-field for proteins (57) and the XOL3 force-field for RNA $(58,59)$, were used to describe the biomolecules during the simulations. A two steps energy-minimization (each with 10,000 cycles) was performed with and 
without restraints, respectively, followed by heating, density equilibration, and short runs of equilibration. The heating was done from $100 \mathrm{~K}$ to $300 \mathrm{~K}$ for 500 picoseconds with restraints on the entire structure and the density equilibration was performed for 500 picoseconds, also with restraints on the entire structure. The equilibration of the structures was run for four short rounds. The first three rounds of equilibration were run for 200 picoseconds each with the main chain atoms constrained. The final round of equilibration was performed for 2 nanoseconds, followed by the production run for 100 nanoseconds. Sander was used for performing the minimization steps and the following steps were performed using the CUDA version of PMEMD from the Amber program suite $(60,61)$.

\section{Confocal microscopy}

Non-adherent K562 cells were counted and collected in $1.5 \mathrm{~mL}$ Eppendorf tubes at 30,000 cells/tube. After a wash, cells were re-suspended in $200 \mu \mathrm{L}$ of phenol-red free media containing $5 \%$ FCS and $N$ terminally fluorescein-isothiocyanate-labeled peptides ( $c$ (peptide) $=1 \mu \mathrm{M}, t=30$ minutes) in the Eppendorf tubes at $37^{\circ} \mathrm{C}$ with vertical rotation to avoid cell pelleting. After incubation, cells were washed once with phenol-red free media, re-suspended again in the same media, and transferred into the wells of an 8-chamber $\mu$-slide (Ibid $I)$. Cells were immediately imaged e at $37^{\circ} \mathrm{C}$ with an SP5 Laser Scanning Confocal Microscope (Leica).

\section{Dicer cleavage assay}

To assess the inhibitory ability of stapled peptides B3 and D1 for pre-miR-21 Dicer cleavage, $10 \mu \mathrm{L}$ solutions of each $N$-terminally acetylated peptide at varying concentrations $(C=10,50,150,300$ or $600 \mathrm{nM}$ ) were prepared with ds pre-miR-21 ( $c=40 \mathrm{nM}$ ) in a buffer of $20 \mathrm{mM}$ Tris hydrochloride (Tris. $\mathrm{HCl}$ ), $12.5 \mathrm{mM} \mathrm{NaCl}, 2.5 \mathrm{mM} \mathrm{MgCl}_{2}, 1 \mathrm{mM}$ dithiothreitol (DTT), $2 \%$ DMSO, and $0.02 \%$ bovine serum albumin (BSA) $(\mathrm{pH}=6.8)$. Next, RhDicer ( $0.5 \mathrm{U} / \mu \mathrm{L}$ solution; Genlantis) was diluted in a buffer of $20 \mathrm{mM}$ Tris. $\mathrm{HCl}, 100 \mathrm{mM} \mathrm{NaCl}, 1 \mathrm{mM} \mathrm{MgCl}$, $1 \mathrm{mM} \mathrm{DTT}, 5 \mathrm{mM} \beta$-mercaptoethanol, $10 \%$ glycerol, and $0.1 \%$ Triton- $\mathrm{X} 100(\mathrm{pH}=6.8)$ to a concentration of $40 \mathrm{U} / \mathrm{mL}$ and then added to the peptide/RNA solutions (final RhDicer concentration: $4 \mathrm{U} / \mathrm{mL}$ ). The reaction solution was incubated for 150 minutes at $37^{\circ} \mathrm{C}$, then denatured for $5 \mathrm{~min}$ at $70^{\circ} \mathrm{C}$, and stored at $4{ }^{\circ} \mathrm{C}$. Subsequently, a $1: 10$ dilution in $\mathrm{H}_{2} \mathrm{O}$ of the reaction solution was performed and added to a Poly $(\mathrm{A})$ Tailing and reverse transcription solution as reported by Cirera et al.(62). The samples were incubated at $42{ }^{\circ} \mathrm{C}$ for 1 hour before subsequent inactivation for 5 minutes at $95^{\circ} \mathrm{C}$ and cooling to $4{ }^{\circ} \mathrm{C}$. Next, qRT-PCR of each sample was performed using the primers miR21-5p-RV1 (GGTCCAGTTTTTTTTTTTTTTTCAAC), miR21-5pFW1 (GCAGTAGCTTATCAGACTGATG), and miRNA-RT1 (CAGGTCCAGTTTTTTTTTTTTTTTVN) in a StepOnePlus ${ }^{\text {TM }}$ real-time PCR system (Thermo Fisher Scientific). Briefly, $1 \mu \mathrm{L}$ of template DNA solution was added to a solution of Luna ${ }^{\circledR}$ Universal Probe qPCR Master Mix (New England Biolabs) with a final primer concentration of $125 \mathrm{nM}$ in $20 \mu \mathrm{L}$ per sample. Samples were initially denatured for 5 minutes at $95^{\circ} \mathrm{C}$ followed by 45 temperature cycles of 15 seconds at $95^{\circ} \mathrm{C}$ and 30 seconds at $66^{\circ} \mathrm{C}$. The quantification was done using miR-21-5p standard curves. To visualize the inhibitory potential of each peptide the normalized abundance of mature miR-21 / \% is plotted against the peptide concentration $c$ (peptide) / nM. 


\section{RESULTS}

\section{Design of TAV2b-derived stapled peptides}

The TAV2b protein consists primarily of a helix-loop-helix motif which binds double-stranded (ds) A-form RNA in a hook-like manner (Supplementary Figure S1)(37). Both helices sit within the RNA major groove with the majority of contacts formed with RNA backbone resulting in the sequenceindependent recognition of ds RNA structures (37). In the crystal structure, TAV2b is bound to a ds palindromic RNA (pal-RNA; single strand sequence: AGACAGCAUUAUGCUGUCUUU) which was also used in our subsequent binding studies. We aimed to exploit the unique structural properties of TAV2b to generate RNA-targeting peptides. To identify the minimal peptide sequence of TAV2b, we synthesized a series of truncated fragments containing both helices but with different lengths (Supplementary Figure S2). The affinity of each fluorescein-isothiocyanate-labeled truncate was then assessed using an electrophoretic mobility shift assay (EMSA). From the truncation series, a 33-mer peptide (wt33, orange, Figure 1A) ranging from K21 to R53 was the shortest analog to retain RNAbinding (Supplementary Figure S2). Isothermal titration calorimetry (ITC) was next used to study the interaction of an unlabeled version of wt33 with ds pal-RNA, revealing moderate affinity $\left(K_{d}=1.19 \mu \mathrm{M}\right.$; Figure 1B). The affinity is about 16-fold lower than the entire RNA-binding domain of TAV2b (amino acids $\left.1-69, K_{d}=75 \mathrm{nM}\right)(37)$. The reported enthalpy-driven binding behavior of TAV2b was also observed for wt33 $\left(\Delta H=-50 \mathrm{kcal} \cdot \mathrm{mol}^{-1}\right)$. Subsequently, circular dichroism (CD) spectroscopy was used to characterize unbound peptide wt33 and pal-RNA. The spectra indicate the expected doublehelical structure for pal-RNA (gray line, Figure 1C) but a mainly unstructured character for unbound wt33 (black line). Co-incubation of pal-RNA and wt33 (orange line, pal-RNA + wt33) resulted in a pronounced negative ellipticity between $\lambda=208$ and $\lambda=222 \mathrm{~nm}$. Notably, this is not the case when the individual spectra of wt33 and pal-RNA are simply added (black dashed line, [pal-RNA + wt33], Figure 1C). The spectral differences between the unbound and bound wt33 suggest that the peptide experiences an increase in $\alpha$-helicity upon RNA-binding.

To stabilize the helical conformation of the unbound peptide and thereby increase its binding affinity a series of wt33-derived hydrocarbon-stapled peptides were designed. Hydrocarbon-stapling involves the introduction of two olefin-bearing, non-natural $\alpha$-methylated amino acids ( $S_{5}$ or $R_{8}$, Supplementary Figure S3) during solid-phase synthesis (43). The preferred spacing is $i, i+4$ and $i, i+7$ aiming at amino acid positions that are not directly involved in target binding (35). The olefin side chains are crosslinked via ring-closing metathesis providing macrocyclic peptides (Supplementary Figure S4). Based on the crystal structure of TAV2b in complex with the pal-RNA duplex, suitable amino acid positions within the wt33 sequence (orange, Figure 1A) were identified (A24, K27, L31, E35, Q44, E48, and A52). These positions facilitate the introduction of three different crosslinks in the $N$-terminal helix (A1-A3) and two in the $C$-terminal helix (B and $\mathbf{C}$ ). In addition to single-stapled peptides, crosslinks were combined resulting in six double-stapled peptides (B1-B3 and C1-C3; Figure 1D). 
A

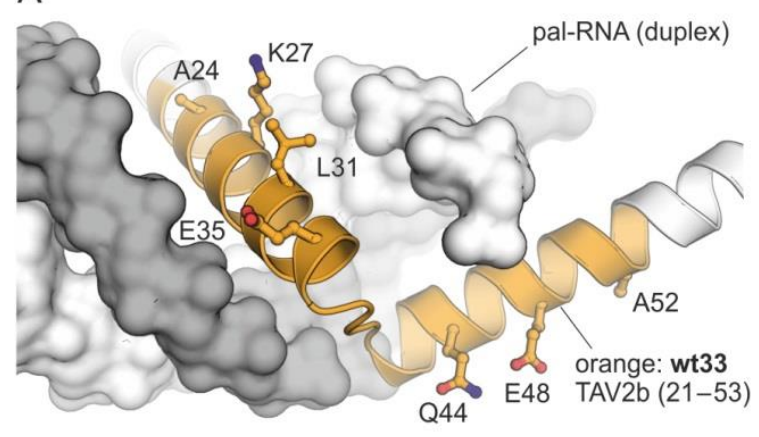

D

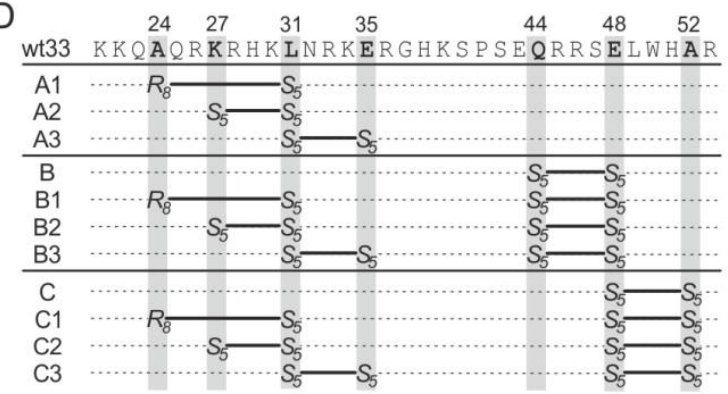

B
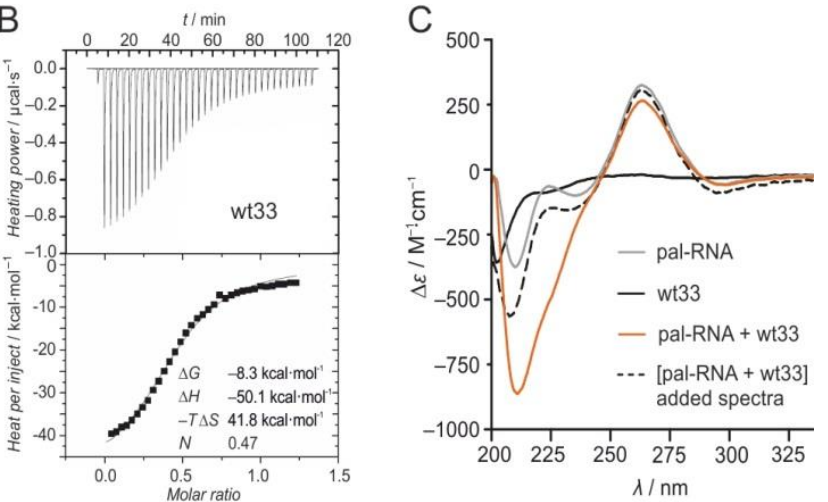

$E$

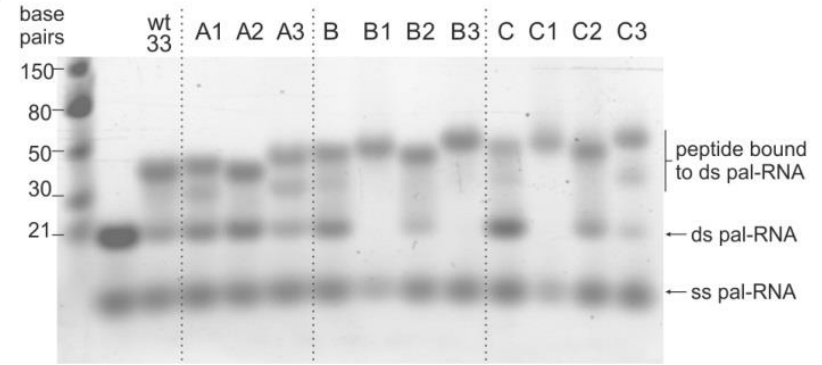

Figure 1. (A) Crystal structure (PDB ID: 2ZI0) highlighting the wt33 sequence (K21-R53, orange, cartoon representation) in complex with ds pal-RNA (gray and white, surface representation). Selected wt33 side chains are shown as ball-and-stick representations. (B) Representative ITC of unlabeled wt33 with the pal-RNA duplex (for full data see Supplementary Table S3 and Figure S5). (C) CD spectra of wt33 ( $c=4 \mu \mathrm{M})$, pal-RNA (c(duplex) $=2 \mu \mathrm{M})$, spectra of pal-RNA (c(duplex) $=2 \mu \mathrm{M})$ with wt33 $(c=4 \mu \mathrm{M})$ in solution (pal-RNA + wt33), and the sum of the two individual spectra ([pal-RNA + wt33]). Buffer: $10 \mathrm{mM}$ sodium phosphate $(\mathrm{pH}=7.4), 100 \mathrm{mM} \mathrm{NaCl}$. (D) Sequences of TAV2b-derived peptides highlighting macrocyclization points. (E) EMSA of ds pal-RNA incubated with fluorescein-isothiocyanate-labeled TAV2b-derived peptides (A1-A3, B-B3, C-C3). Experiments employed $10 \%$ native PAGE $(c(\mathrm{RNA})=3 \mu \mathrm{M}, c$ (peptide) $=6 \mu \mathrm{M})$. Running buffer: $25 \mathrm{mM}$ Tris $(\mathrm{pH}=8.0), 200 \mathrm{mM}$ glycine, $20 \mathrm{mM} \mathrm{NaCl}$. For full gels including fluorescein readout see Supplementary Figure S6 (ds = double-stranded, ss $=$ single-stranded).

Eleven stapled peptides were synthesized and their binding to ds pal-RNA assessed via EMSA (Figure 1E and Supporting Figure S6). Here, pal-RNA mainly appeared as a band corresponding to the ds structure. Formation of the peptide/RNA complex is indicated by the disappearance of the palRNA duplex band and the appearance of a new elevated band. Peptides were labeled with fluorescein-isothiocyanate (FITC) as a means to verify the formation of such peptide/RNA complexes using fluorescence visualization (Supplementary Figure S6). Due to their positive charge unbound peptides did not migrate into the gel. Elevated bands, indicative of RNA binding, were observed for all peptides. The disappearance of free ds pal-RNA was only observed for the double-stapled peptides B1, B3, and C1 hinting at particularly potent RNA-binding.

\section{Double-stapled peptides show enhanced helicity and affinity for ds pal-RNA}

ITC binding experiments with unlabeled analogs of B1, B3, and C1 reveal 4.6-, 17.0-, and 6.0-fold increased affinity, when compared to linear peptide wt33 (Figure 2A). In analogy to wt33, all three stapled peptides exhibit enthalpy-driven binding, though to a reduced extent $(\Delta H(\mathbf{w t} 33)=5.0$ vs. $\Delta H$ (stapled peptides $\left.)=3.9-4.1 \mathrm{kcal} \cdot \mathrm{mol}^{-1}\right)$. This decrease in binding enthalpy is compensated by a 
reduction in the entropic penalty upon binding $(-T \cdot \Delta S(\mathbf{w t 3 3})=42$ vs. $-T \cdot \Delta S$ (stapled peptides $)=30-32$ $\mathrm{kcal} \cdot \mathrm{mol}^{-1}$ ) which likely stems from the helical pre-organization induced by stapling. Evidence of this structural pre-organization was also observed with CD spectroscopy. In solution, peptides B1, B3, and $\mathbf{C} 1$ display characteristic $\alpha$-helical spectra. Unlike wt33, an enhancement in ellipticity between $\lambda=$ 208 and $\lambda=222 \mathrm{~nm}$ was not observed when these peptides were incubated with ds pal-RNA (Supplementary Figures S7-S9). These data indicate that stapling indeed enforced the $\alpha$-helical conformation of peptides B1, B3, and C1, thereby promoting pal-RNA duplex binding. For subsequent experiments, control peptide D1 was included which features two hydrocarbon crosslinks within the RNA-interface and does not bind to ds pal-RNA in EMSAs (Supplementary Table S2 and Figure S10). Initially, the entire FITC-labeled peptide panel including D1 was characterized via CD spectroscopy to determine $\alpha$-helicity in the unbound form (Figure 2B). Notably, all stapled peptides showed higher $\alpha$-helicity than wt33 $(\alpha$-helicity $=7.3 \%$ ) with the double-stapled derivatives exhibiting a stronger helical character than the mono-stapled analogs (Figure 2B). High-affinity peptides B1, B3, and C1 are among the most helical sequences ( $\alpha$-helicity $=82-85 \%$ ) with their CD spectra showing minima at $\lambda=208 \mathrm{~nm}$ and $\lambda=222 \mathrm{~nm}$ characteristic for $\alpha$-helices (Figure 2C).
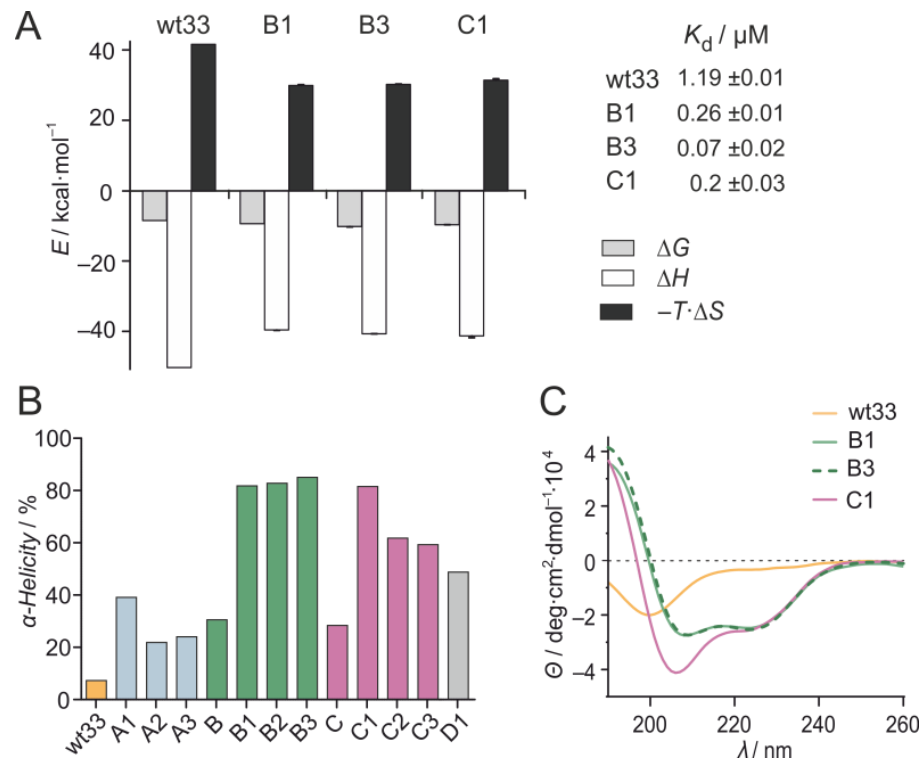

Figure 2. (A) Thermodynamic profiles of unlabeled wt33, B1, B3, and C1 as determined by ITC, including $K_{d^{-}}$ values (measurements were performed in triplicate, errors account for $1 \sigma$; for full data see Supplementary Table S3 and Figure S11-S13). (B) Percentage of $\alpha$-helical secondary structure content for each FITC-labeled peptide (for complete secondary structure distribution see Supplementary Table S4). (C) Overlaid CD spectra of FITClabeled wt33, B1, B3, and C1 ( $($ peptide $)=75 \mu \mathrm{M})$. Buffer: $10 \mathrm{mM}$ sodium phosphate $(\mathrm{pH}=7.4)$.

\section{Peptide stapling increases protease stability and cell permeability}

A protease stability assay was conducted by incubating the FITC-labeled peptides with proteinase $\mathrm{K}$ and monitoring peptide integrity over time by HPLC-MS to determine peptide half-life ( $t_{1 / 2}$, Figure $3 A)(47)$. Linear peptide wt33 experienced rapid cleavage $\left(t_{1 / 2}=2.4\right.$ minutes, Supplementary Table S5), whereas the introduction of crosslinks in the $C$-terminal helix resulted in increased stability (B and $\mathbf{C}, t_{1 / 2}=65$ and 78 minutes, respectively) while stapling of the $N$-terminal helix did not improve 
protease resistance ( $\mathbf{A} 1-\mathbf{A} 3, t_{1 / 2} \approx 3$ minutes). A combination of both $\mathrm{N}$ - and $\mathrm{C}$-terminal staples yielded the most stable peptides, with double-stapled peptides $\mathbf{B} 1$ and $\mathbf{C} 1$ experiencing the strongest increase in tolerance towards proteinase K (B1: 354-fold and C1: 265-fold increased stability compared to wt33, Figure 3B).
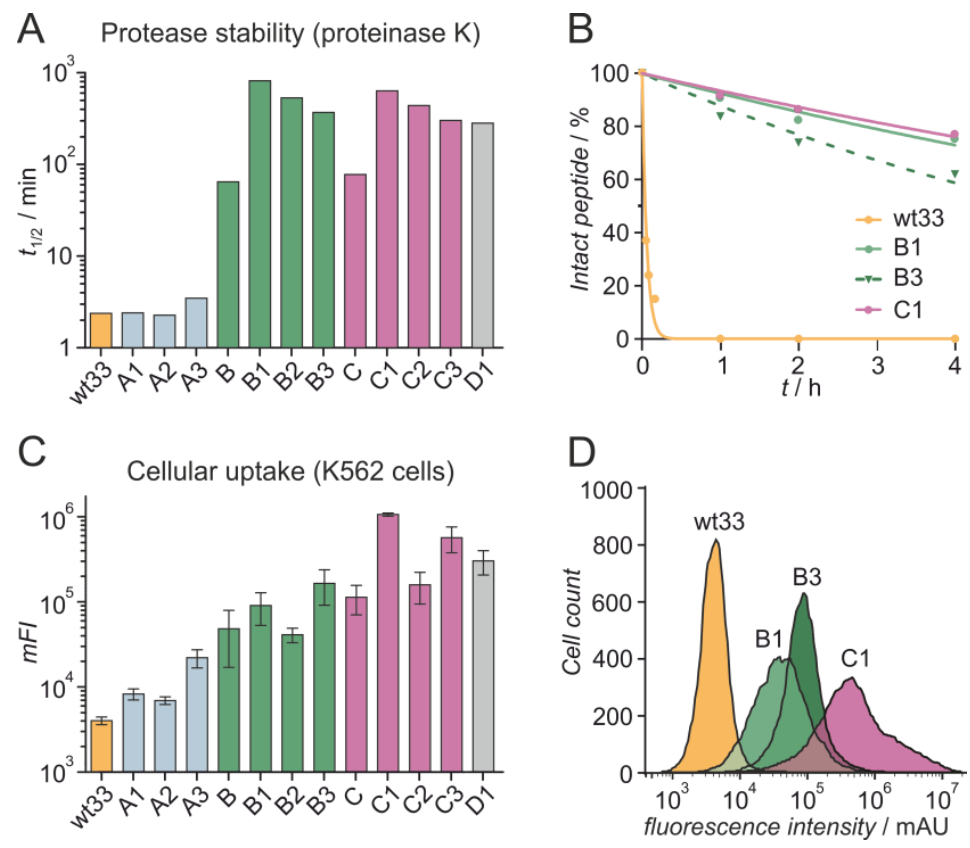

Figure 3. (A) Half-life $\left(t_{1 / 2}\right)$ values for each fluorescently-labeled peptide $(C=50 \mu \mathrm{M})$ in the presence of proteinase $\mathrm{K}\left(c=10 \mu \mathrm{g} \cdot \mathrm{mL}^{-1}\right)$. Buffer: $10 \mathrm{mM}$ Tris $(\mathrm{pH}=8.0), 150 \mathrm{mM} \mathrm{NaCl}$. (B) Percentage of intact fluorescently-labeled peptide remaining over time in the presence of proteinase K. (C) $\mathrm{mFI}$ values of $\mathrm{K} 562$ human leukemia cells treated with fluorescently-labeled derivatives of each peptide ( $c=1 \mu \mathrm{M}, t=90$ minutes). (D) Fluorescence intensity distribution of K562 cells exposed to fluorescently-labeled wt33, B1, B3, and C1 (triplicate of measurements, errors account for $1 \sigma)$.

The cellular uptake of the peptides was assessed using K562 human leukemia cells and flow cytometry as readout (63). Cells were treated with FITC-labeled peptides ( $c=1 \mu \mathrm{M}, t=90 \mathrm{~min}$ ) which was followed by a stringent wash protocol including tryptic digestion. Flow cytometry-based mean fluorescence intensities ( $m F l$ ) were then determined (Figure 3C). Compared to wt33 ( $\mathrm{mFI}=4031 \mathrm{AU}$ ) all stapled peptides caused increased fluorescence intensities indicative of increased cellular uptake. In line with trends observed for $\alpha$-helicity (Figure 2B) and proteolytic stability (Figure 3A), the doublestapled peptides displayed an increased cellular uptake when compared to their mono-stapled analogs. Overall, double-stapled peptide $\mathbf{C} 1$ showed the highest increase in uptake relative to wt33 (264-fold; Figure 3D). Despite its lower ds RNA affinity, considerably enhanced cellular uptake was also observed for control peptide D1. Based on these observations and their high affinity for ds palRNA, we considered the use of double-stapled peptides B3, C1 and control D1 for further investigations. For that reason, cellular uptake of the peptides was also investigated by live cell confocal microscopy confirming their cell permeability (Supplementary Figure S14). 


\section{Double-stapled peptides bind ds miR-21 structures}

The protein TAV2b was reported to preferably bind ds RNA and we therefore assessed the binding of B3 and $\mathbf{C} 1$ to alternative nucleic acid structures using EMSA. Analog to TAV2b, both stapled peptides did not bind single-stranded RNA (ss RNA) or the ds DNA analog of pal-RNA (Supplementary Figures S15 and S16). Notably, we also did not observe binding of B3 and $\mathbf{C} 1$ to a mixture of yeast transfer RNAs (t-RNAs) indicating that peptide binding indeed require accessible RNA duplex structures. Although not reported for TAV2b, other viral suppressors of RNA silencing are known to bind microRNAs (miRNAs) (64). Similar to siRNAs, miRNAs are short non-coding RNAs that are incorporated into the RISC complex leading to the blockage or degradation of complement mRNA (65). MiRNAs arise from long stem-loop-bearing RNAs in the nucleus (pri-miRNAs) which are processed and exported as shorter hairpin structures, so-called pre-miRNAs. Pre-miRNAs are further processed by the endoribonuclease Dicer resulting in the formation of mature ds miRNAs (66).

Given the sequence-independent nature of TAV2b-binding, we suspected double-stapled peptides B3 and $\mathbf{C} 1$ to also bind miRNAs. To test this hypothesis, ds miRNA-21 (miR-21) was chosen as an example. Similar to pal-RNA, the miR-21 duplex is comprised of two short RNA strands but features a central one-nucleotide bulge as well as a single mismatched base pair (black, Figure $4 A)(67,68)$. In pre-miR-21, the two RNA strands (black) are connected by a partially selfcomplementary sequence (gray, Figure 4A) resulting in the adaption of a hairpin-like structure. Initially, EMSAs were used to investigate the ability of FITC-labeled B3, C1, and D1 to bind ds miR-21. While we observed the formation of peptide/RNA complexes for B3 and C1, control peptide D1 did not show binding (Supplementary Figure S17-S19). It has been reported that the binding of ligands can increase the stability of nucleic acid duplexes (69). Therefore, thermal denaturation experiments were performed to test the effect of peptide binding on the stability of the miR-21 duplex. Indeed, the incubation of miR-21 $(c=2 \mu \mathrm{M})$ with unlabeled peptides B3 or $\mathbf{C 1}(c=4 \mu \mathrm{M})$ resulted in increased melting temperatures $\left(\Delta T_{\mathrm{m}}>10^{\circ} \mathrm{C}\right)$, while D1 only showed a mild effect on duplex stability (Figure 4B).

Having confirmed ds miR-21-binding of B3 and C1, we assessed their binding to endogenous miR-21 in a cellular context. For that purpose pull-down experiments were performed in which K562 human leukemia cells were initially incubated with biotinylated analogs of B3, C1, or D1 ( $c=10 \mu \mathrm{M}, t$ $=180$ minutes). Subsequently, cells were rigorously washed, lysed, and finally lysates were treated with streptavidin beads to capture the peptides in complex with RNA. After extraction of RNA from the beads, the amount of miR-21 was evaluated by quantitative reverse transcription polymerase chain reaction (qRT-PCR). In this experimental setup, peptide cell permeability, bio-stability, and RNAbinding contribute to pull-down efficiency. For peptides B3 and C1 we observed enrichment when compared to the control peptide D1 (Figure 4C), which was particularly pronounced for B3 (35-fold). Given the structural similarities between ds miR-21 and its hairpin precursor, pre-miR-21 (Figure 4A), we also tested binding of FITC-labeled B3 and $\mathbf{C} 1$ to pre-miR-21 initially using EMSA. For both double-stapled peptides, EMSA indicated pre-miRNA-binding (Supplementary Figures S17 and S18). In pull-down experiments, however, we only observed enrichment of pre-miR-21 when using biotinylated B3 (4-fold, Figure 4D), though to a lower extent than miR-21 (35-fold, Figure 4C). In line 
with its lower miR-21 pull-down efficiency, $\mathbf{C} 1$ did not show detectable pre-miR-21 enrichment. Based on the good performance of B3 in the miR-21 and pre-miR-21 pull-down experiments, this doublestapled peptide was selected for further investigations.

A
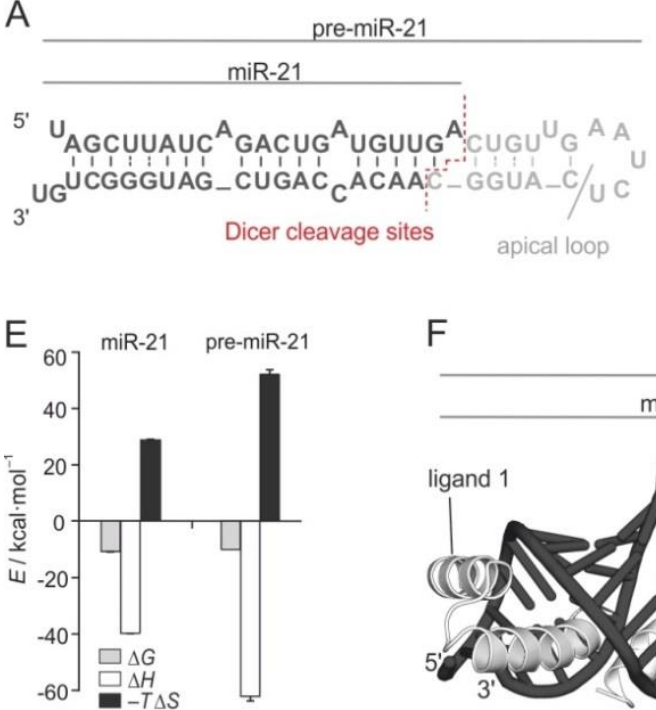

F
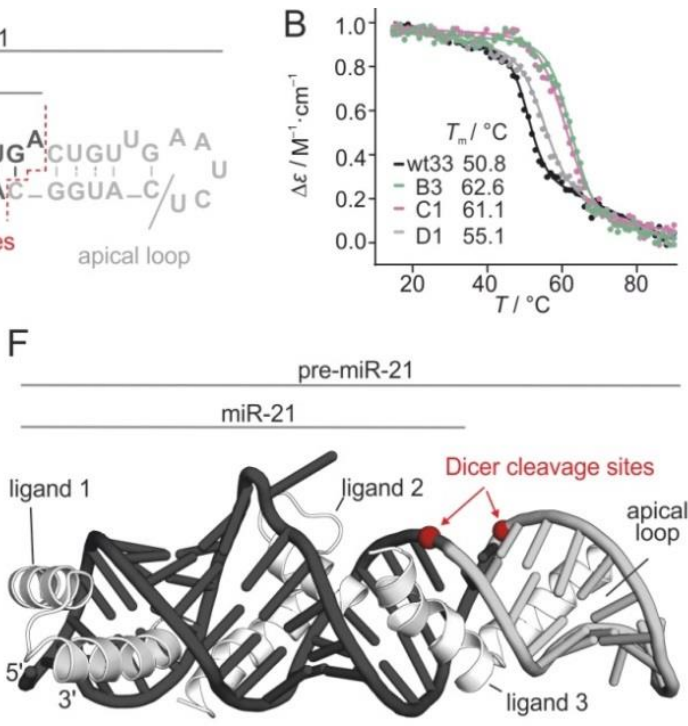
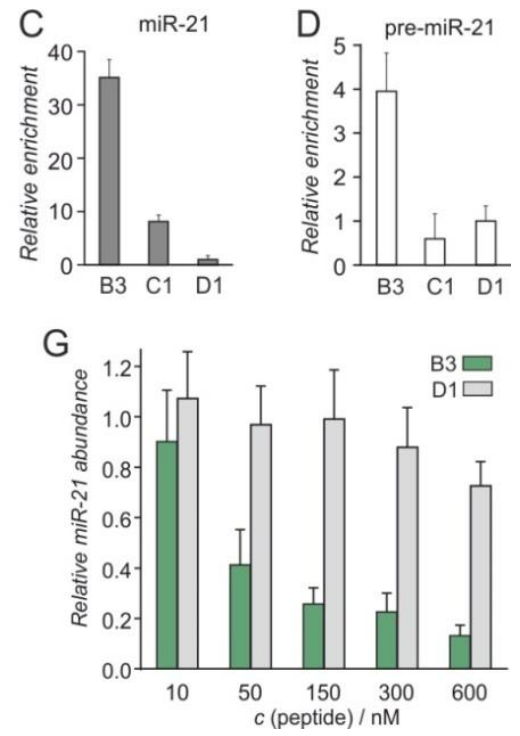

Figure 4. (A) Sequence of miR-21 (black) and hairpin structure pre-miR-21 (black/gray). Locations of the Dicer cleavage sites are indicated (red). (B) Melting profiles of miR-21 in absence and presence of unlabeled doublestapled peptides B3, C1, and D1, respectively $(\lambda=267 \mathrm{~nm}, c(\mathrm{miR}-21)=2 \mu \mathrm{M}, c($ peptide $)=4 \mu \mathrm{M}$. Buffer: $10 \mathrm{mM}$ sodium phosphate ( $\mathrm{pH}=7.4), 100 \mathrm{mM} \mathrm{NaCl})$. (C/D) Enrichment of miR-21 and pre-miR-21, respectively, following treatment of K562 cells with the biotinylated peptides B3, C1, and D1 as well as streptavidin-mediated selection of peptide-bound RNA relative to total RNA after cell lysis (triplicate of measurements, errors account for $1 \sigma$ ). (E) ITC-based thermodynamic profiles of acetylated B3 binding to miR-21 and pre-miR-21 (measurements were performed in triplicate; for full data see Supplementary Table S3 and Figures S20 and S21). (F) SimRNP model of a complex involving three wt33 peptides (white, cartoon representation) and pre-miR-21 (black and gray, cartoon representation). Coordinates of the model can be found as supplementary data. Locations of the Dicer cleavage sites are indicated (red). (G) Relative miR-21 abundance after Dicer processing of pre-miR-21 in the presence and absence of acetylated B3 or D1 using qRT-PCR (relative to absence of peptide control; $c\left(\right.$ pre-miR-21) $=40 \mathrm{nM}, c($ Dicer $)=4 \mathrm{U} \cdot \mathrm{mL}^{-1}$; triplicate of measurements, errors account for $\left.1 \sigma\right)$.

Having qualitatively assessed B3 binding to ds miR-21 and pre-miR-21, we next investigated complex formation using ITC (Figure 4E and Supplementary Figure S20) with acetylated peptide versions. For the B3/miR-21 complex ITC revealed a very high stability $\left(K_{d}=18 \pm 2 \mathrm{nM}\right)$ exceeding the binding affinity of B3 for the pal-RNA duplex ( $K_{d}=70 \pm 1 \mathrm{nM}$, Supplementary Table S3). The binding process was again enthalpy-driven with the expected binding stoichiometry of about 2:1 (B3/miR-21, $N=0.43$ ). We also used ITC to investigate B3 binding to pre-miR-21, revealing a high affinity $\left(K_{d}=75 \pm 8 \mathrm{nM}\right)$ though with a changed binding stoichiometry indicative of 3:1 binding (B3/pre-miR-21, $N=0.28$; Supplementary Table S3). Given the elongated stem region in pre-miR-21 we suspected the binding of an additional B3 molecule. To test the general feasibility of such a complex involving one RNAhairpin and three peptides, replica-exchange Monte Carlo simulations of the unmodified peptide sequence with pre-miR-21 were performed using SimRNA and SimRNP, computational methods that 
allow for flexible modeling of protein/RNA complexes (51). According to the simulation, pre-miR-21 can indeed accommodate up to three peptide sequences (Figure 4F), two of them (ligand 1 and 2) in the area of the miR-21 duplex region (black) while the third peptide (ligand 3) mainly contacts the apical loop (gray, Figure 4F). Notably, ligand 3 is predicted to bind the region that is recognized by the endoribonuclease Dicer.

Dicer binding of pre-miR-21 eventually results in strand cleavage at two sites (C30 and G47; red, Figure 4F) yielding mature ds miR-21 (black, Figure 4F). Based on the presumably overlapping binding sites of our peptides and Dicer, we were interested to test how the high-affinity acetylated double-stapled peptide B3 affects pre-miR-21 maturation. For this purpose a biochemical maturation assay was established where recombinant $\operatorname{Dicer}\left(c=4 \mathrm{U} \cdot \mathrm{mL}^{-1}\right)$ was used to process pre-miR-21 ( $c=$ $40 \mathrm{nM}$ ) into mature miR-21. A qRT-PCR detection protocol (62) was adapted to monitor miR-21 formation. Notably, pre-miR-21 maturation by Dicer was inhibited in a concentration-dependent manner by double-stapled peptide B3 (green, Figure 4G) while control peptide D1 (gray, Figure 4G) showed only small effects. Half-maximal Dicer inhibition by B3 is about $50 \mathrm{nM}$ which is in the concentration range of pre-miR-21 $(c=40 \mathrm{nM})$ in this assay. This indicates efficient competition with Dicer under these conditions.

\section{DISCUSSION}

The design of RNA-targeting ligands remains an outstanding challenge. Antisense oligonucleotides represent the most established entities and while possessing high affinity and selectivity they tend to suffer from delivery issues (70). Alternative approaches to target RNA make use of extended or multivalent ligands derived from natural products such as aminoglycosides $(18,71,72)$. However, these approaches involve complicated development processes since only limited structural information is available. With the growing number of strategies to derive protein mimetics (73), new avenues towards the design of potent nucleic acid binders are being explored (74-77). Herein, we applied a structure-based design approach to derive constrained peptide mimetics of the RNA-binding protein TAV2b. As an initial starting point, we identified a 33-mer TAV2b-derived peptide (wt33) as a minimal sequence for ds siRNA binding. Based on this peptide, a panel of hydrocarbon-stapled peptides was designed aiming at the stabilization of the RNA-bound $\alpha$-helical conformation of TAV2b. The obtained stapled peptides show enhanced $\alpha$-helicity, protease stability, and cell permeability, particularly pronounced for double-stapled analogs. From that panel, double-stapled peptide B3 was investigated in most detail. It binds palindromic siRNA with high affinity but not single-stranded, transfer RNA or ds DNA. Notably, B3 also shows high affinity for additional double-stranded RNA structures including microRNA-21 and its precursor pre-miR-21. Binding of B3 to pre-miR-21 was also shown to inhibit RNA maturation by the endoribonuclease Dicer in a biochemical assay.

Double-stapled peptide B3, represents a structural mimetic of the RNA-binding TAV2b protein. Recapitulating central features of TAV2b binding, B3 engages ds RNA in a sequence-independent and enthalpy-driven manner. In analogy to TAV2b, complex formation with ds RNA is strongly preferred over ss RNA, likely due to the absence of a defined binding groove in the unpaired and highly flexible ss RNA chain. B3 also exhibits a strong preference for ds RNA over ds DNA. This can 
be explained as ds DNA tends to exist in the B-form possessing wider and shallower major grooves than A-form RNA thereby preventing analogous interaction patterns (78). The $\alpha$-helical conformation of TAV2b is central to its high affinity for ds RNA. Our studies indicate that a truncated version of TAV2b (wt33) is unstructured in its free form but shows high $\alpha$-helicity upon binding. This can be expected to result in a considerable entropic penalty and likely contributes to wt33's relatively low affinity. Introduction of chemical constraints to stabilize the $\alpha$-helical conformation, as seen for B3, consequently increases binding affinity for ds RNA.

Overall, our results highlight that RNA-binding proteins represent a valuable starting point for the structure-based design of cell-permeable, high affinity RNA ligands, in particular when considering the growing number of available protein:RNA complex structures (8). Such design strategies can complement established protein- and nucleic acid-based targeting approaches as well as combinatorial screening efforts $(17,70,79,80)$. Additionally, peptide-based ligands can offer new opportunities to develop probes with fine-tuned or switchable RNA-binding characteristics. The double-stapled peptide B3 is a prime example of a modular scaffold combining bioavailability and stability with high affinity binding. With this, B3 represents an attractive starting point for the future design of ligands with altered RNA-recognition properties.

\section{SUPPLEMENTARY DATA}

Supplementary data are can be found on ChemRxiv. Coordinates of the model shown in Figure 4F are available upon request.

\section{ACKNOWLEDGEMENTS}

We thank the group of Prof. Roland Winter (TU Dortmund) for access to the CD spectrometer. We also thank Dr. Alan Gerber and Mathias Wendt (VU Amsterdam) for helpful discussions and critical reading of this manuscript.

\section{FUNDING}

This work was supported by the European Research Council (ERC starting grant number 678623) and by AstraZeneca, Bayer CropScience, Bayer HealthCare, Boehringer Ingelheim, Merck KGaA, and the Max Planck Society. C.N. and J.M.B. were supported by the Polish National Science Center (NCN, grant 2017/26/A/NZ1/01083 to J.M.B.). S.M. was supported by IIMCB statutory funds. Computational analyses were performed using the resources of IIMCB, the Poznan Supercomputing and Networking Center at the Institute of Bioorganic Chemistry, Polish Academy of Sciences (grant: 312), the Polish Grid Infrastructure (grants: rnpmd, rnpmc, simcryox), and the Interdisciplinary Centre for Mathematical and Computational Modelling at the University of Warsaw (grant: GB76-30). 


\section{REFERENCES}

1. tenOever,B.R. (2016) The Evolution of Antiviral Defense Systems. Cell Host Microbe, 19, 142-149.

2. Ding,S.-W. (2010) RNA-based antiviral immunity. Nat. Rev. Immunol., 10, 632-644.

3. Haasnoot,J., Westerhout,E.M. and Berkhout,B. (2007) RNA interference against viruses: strike and counterstrike. Nat. Biotechnol., 25, 1435-1443.

4. Guo,Z., Li,Y. and Ding,S.-W. (2019) Small RNA-based antimicrobial immunity. Nat. Rev. Immunol., $19,31-44$.

5. Daròs,J.-A. (2017) Viral suppressors: Combatting RNA silencing. Nat. Plants, 3, 17098.

6. Csorba,T., Kontra,L. and Burgyán,J. (2015) viral silencing suppressors: Tools forged to fine-tune host-pathogen coexistence. Virology, 479-480, 85-103.

7. Hentze,M.W., Castello,A., Schwarzl,T. and Preiss,T. (2018) A brave new world of RNA-binding proteins. Nat. Rev. Mol. Cell Biol., 19, 327-341.

8. Krüger,D.M., Neubacher,S. and Grossmann,T.N. (2018) Protein-RNA interactions: structural characteristics and hotspot amino acids. RNA, 24, 1457-1465.

9. Rauch,S., He,E., Srienc,M., Zhou,H., Zhang,Z. and Dickinson,B.C. (2019) Programmable RNAGuided RNA Effector Proteins Built from Human Parts. Cell, 178, 122-134.e12.

10. Chen,Y., Yang,F., Zubovic,L., Pavelitz,T., Yang,W., Godin,K., Walker,M., Zheng,S., Macchi,P. and Varani,G. (2016) Targeted inhibition of oncogenic miR-21 maturation with designed RNAbinding proteins. Nat. Chem. Biol., 12, 717-723.

11. Adamala,K.P., Martin-Alarcon,D.A. and Boyden,E.S. (2016) Programmable RNA-binding protein composed of repeats of a single modular unit. Proc. Natl. Acad. Sci., 113, E2579-E2588.

12. Choudhury,R., Tsai,Y.S., Dominguez,D., Wang,Y. and Wang,Z. (2012) Engineering RNA endonucleases with customized sequence specificities. Nat. Commun., 3, 1147.

13. Connelly,C.M., Moon,M.H. and Schneekloth,J.S. (2016) The Emerging Role of RNA as a Therapeutic Target for Small Molecules. Cell Chem. Biol., 23, 1077-1090.

14. Donlic,A. and Hargrove,A.E. (2018) Targeting RNA in mammalian systems with small molecules. Wiley Interdiscip. Rev. RNA, 9, e1477.

15. Warner,K.D., Hajdin,C.E. and Weeks,K.M. (2018) Principles for targeting RNA with drug-like small molecules. Nat. Rev. Drug Discov., 17, 547-558.

16. L Garner,A. (2019) RNA-targeted drug discovery: moving beyond promiscuous small-molecule scaffolds. Future Med. Chem., 11, 2487-2490.

17. Gareiss,P.C., Sobczak,K., McNaughton,B.R., Palde,P.B., Thornton,C.A. and Miller,B.L. (2008) Dynamic combinatorial selection of molecules capable of inhibiting the (CUG) repeat RNAMBNL1 interaction in vitro: Discovery of lead compounds targeting myotonic dystrophy (DM1). J. Am. Chem. Soc., 130, 16254-16261.

18. Yan,H., Bhattarai,U., Guo,Z.-F. and Liang,F.-S. (2017) Regulating miRNA-21 Biogenesis By Bifunctional Small Molecules. J. Am. Chem. Soc., 139, 4987-4990.

19. Rzuczek,S.G., Colgan,L.A., Nakai,Y., Cameron,M.D., Furling,D., Yasuda,R. and Disney,M.D. (2017) Precise small-molecule recognition of a toxic CUG RNA repeat expansion. Nat. Chem. Biol., 13, 188-193. 
20. Penas,C., Mascareñas,J.L. and Vázquez,M.E. (2016) Coupling the folding of a $\beta$-hairpin with chelation-enhanced luminescence of $\mathrm{Tb}$ (iii) and $\mathrm{Eu}$ (iii) ions for specific sensing of a viral RNA. Chem. Sci., 7, 2674-2678.

21. McColl,D.J., Honchell,C.D. and Frankel,A.D. (1999) Structure-based design of an RNA-binding zinc finger. Proc. Natl. Acad. Sci. U. S. A., 96, 9521-9526.

22. Harrison,R.S., Shepherd,N.E., Hoang,H.N., Beyer,R.L., Ruiz-Gómez,G., Kelso,M.J., Mei Kok,W., Hill,T.A., Abbenante,G. and Fairlie,D.P. (2014) Helical cyclic pentapeptides constrain HIV-1 Rev peptide for enhanced RNA binding. Tetrahedron, 70, 7645-7650.

23. Athanassiou,Z., Dias,R.L.A., Moehle,K., Dobson,N., Varani,G. and Robinson,J.A. (2004)

Structural Mimicry of Retroviral Tat Proteins by Constrained $\beta$-Hairpin Peptidomimetics: Ligands with High Affinity and Selectivity for Viral TAR RNA Regulatory Elements. J. Am. Chem. Soc., 126, 6906-6913.

24. Belashov,I.A., Crawford,D.W., Cavender,C.E., Dai,P., Beardslee,P.C., Mathews,D.H., Pentelute,B.L., McNaughton,B.R. and Wedekind,J.E. (2018) Structure of HIV TAR in complex with a Lab-Evolved RRM provides insight into duplex RNA recognition and synthesis of a constrained peptide that impairs transcription. Nucleic Acids Res., 46, 6401-6415.

25. Bose,D., Nahar,S., Rai,M.K., Ray,A., Chakraborty,K. and Maiti,S. (2015) Selective inhibition of miR-21 by phage display screened peptide. Nucleic Acids Res., 43, 4342-4352.

26. Sakamoto,K., Otake,K. and Umemoto,T. (2017) Discovery of peptidic miR-21 processing inhibitor by mirror image phage display: A novel method to generate RNA binding D-peptides. Bioorg. Med. Chem. Lett., 27, 826-828.

27. Pai,J., Hyun,S., Hyun,J.Y., Park,S.H., Kim,W.J., Bae,S.H., Kim,N.K., Yu,J. and Shin,I. (2016) Screening of Pre-miRNA-155 Binding Peptides for Apoptosis Inducing Activity Using Peptide Microarrays. J. Am. Chem. Soc., 138, 857-867.

28. Pelay-Gimeno,M., Glas,A., Koch,O. and Grossmann,T.N. (2015) Structure-Based Design of Inhibitors of Protein-Protein Interactions: Mimicking Peptide Binding Epitopes. Angew. Chemie Int. Ed., 54, 8896-8927.

29. Robertson,N. and Spring,D. (2018) Using Peptidomimetics and Constrained Peptides as Valuable Tools for Inhibiting Protein-Protein Interactions. Molecules, 23, 959.

30. Azzarito,V., Long,K., Murphy,N.S. and Wilson,A.J. (2013) Inhibition of a-helix-mediated proteinprotein interactions using designed molecules. Nat. Chem., 5, 161-173.

31. Lau,Y.H., de Andrade,P., Wu,Y. and Spring,D.R. (2015) Peptide stapling techniques based on different macrocyclisation chemistries. Chem. Soc. Rev., 44, 91-102.

32. Cromm,P.M., Spiegel,J. and Grossmann,T.N. (2015) Hydrocarbon Stapled Peptides as Modulators of Biological Function. ACS Chem. Biol., 10, 1362-1375.

33. Walensky,L.D. and Bird,G.H. (2014) Hydrocarbon-Stapled Peptides: Principles, Practice, and Progress. J. Med. Chem., 57, 6275-6288.

34. Blackwell,H.E. and Grubbs,R.H. (1998) Highly efficient synthesis of covalently cross-linked peptide helices by ring-closing metathesis. Angew. Chemie - Int. Ed., 37, 3281-3284.

35. Schafmeister,C.E., Po,J. and Verdine,G.L. (2000) An All-Hydrocarbon Cross-Linking System for 
Enhancing the Helicity and Metabolic Stability of Peptides. J. Am. Chem. Soc., 122, 5891-5892.

36. Lucy,A.P., Guo,H.S., Li,W.X. and Ding,S.W. (2000) Suppression of post-transcriptional gene silencing by a plant viral protein localized in the nucleus. EMBO J., 19, 1672-80.

37. Chen,H.-Y., Yang,J., Lin,C. and Yuan,Y.A. (2008) Structural basis for RNA-silencing suppression by Tomato aspermy virus protein 2b. EMBO Rep., 9, 754-60.

38. Cantor,C.R., Warshaw,M.M. and Shapiro,H. (1970) Oligonucleotide interactions. 3. Circular dichroism studies of the conformation of deoxyoligonucleotides. Biopolymers, 9, 1059-1077.

39. Fasman,G.D. (1975) Handbook of Biochemistry and Molecular Biology, Vol. 1: Nucleic Acids 3d ed. CRC Press.

40. Kallansrud,G. and Ward,B. (1996) A Comparison of Measured and Calculated Single- and Double-Stranded Oligodeoxynucleotide Extinction Coefficients. Anal. Biochem., 236, 134-138.

41. Murphy,J.H. and Trapane,T.L. (1996) Concentration and extinction coefficient determination for oligonucleotides and analogs using a general phosphate analysis. Anal. Biochem., 240, 273282.

42. Cavaluzzi,M.J. and Borer,P.N. (2004) Revised UV extinction coefficients for nucleoside-5'monophosphates and unpaired DNA and RNA. Nucleic Acids Res., 32, e13-e13.

43. Kim,Y.-W., Grossmann,T.N. and Verdine,G.L. (2011) Synthesis of all-hydrocarbon stapled $\alpha$ helical peptides by ring-closing olefin metathesis. Nat. Protoc., 6, 761-771.

44. Niklasson,M., Andresen,C., Helander,S., Roth,M.G.L., Zimdahl Kahlin,A., Lindqvist Appell,M. and Lundström,P. (2015) Robust and convenient analysis of protein thermal and chemical stability. Protein Sci., 24, 2055-2062.

45. Poschner,B.C., Reed,J., Langosch,D. and Hofmann,M.W. (2007) An automated application for deconvolution of circular dichroism spectra of small peptides. Anal. Biochem., 363, 306-308.

46. Böhm,G., Muhr,R. and Jaenicke,R. (1992) Quantitative analysis of protein far UV circular dichroism spectra by neural networks. Protein Eng. Des. Sel., 5, 191-195.

47. Cromm,P.M., Spiegel,J., Küchler,P., Dietrich,L., Kriegesmann,J., Wendt,M., Goody,R.S., Waldmann,H. and Grossmann,T.N. (2016) Protease-Resistant and Cell-Permeable DoubleStapled Peptides Targeting the Rab8a GTPase. ACS Chem. Biol., 11, 2375-2382.

48. Piatkowski,P., Kasprzak,J.M., Kumar,D., Magnus,M., Chojnowski,G. and Bujnicki,J.M. (2016) RNA 3D Structure Modeling by Combination of Template-Based Method ModeRNA, TemplateFree Folding with SimRNA, and Refinement with QRNAS. Methods Mol. Biol., 1490, 217-235.

49. Rother,M., Rother,K., Puton,T. and Bujnicki,J.M. (2011) ModeRNA: a tool for comparative modeling of RNA 3D structure. Nucleic Acids Res., 39, 4007-4022.

50. Shortridge,M.D., Walker,M.J., Pavelitz,T., Chen,Y., Yang,W. and Varani,G. (2017) A Macrocyclic Peptide Ligand Binds the Oncogenic MicroRNA-21 Precursor and Suppresses Dicer Processing. ACS Chem. Biol., 12, 1611-1620.

51. Boniecki,M.J., Lach,G., Dawson,W.K., Tomala,K., Lukasz,P., Soltysinski,T., Rother,K.M. and Bujnicki,J.M. (2016) SimRNA: a coarse-grained method for RNA folding simulations and 3D structure prediction. Nucleic Acids Res., 44, e63-e63.

52. Kozomara,A., Birgaoanu,M. and Griffiths-Jones,S. (2019) miRBase: from microRNA sequences to 
function. Nucleic Acids Res., 47, D155-D162.

53. Stasiewicz,J., Mukherjee,S., Nithin,C. and Bujnicki,J.M. (2019) QRNAS: software tool for refinement of nucleic acid structures. BMC Struct. Biol., 19, 5.

54. Boniecki,M., Rotkiewicz,P., Skolnick,J. and Kolinski,A. (2003) Protein fragment reconstruction using various modeling techniques. J. Comput. Aided. Mol. Des., 17, 725-738.

55. Salomon-Ferrer,R., Case,D.A. and Walker,R.C. (2013) An overview of the Amber biomolecular simulation package. Wiley Interdiscip. Rev. Comput. Mol. Sci., 3, 198-210.

56. Sun,Y. and Kollman,P.A. (1995) Hydrophobic solvation of methane and nonbond parameters of the TIP3P water model. J. Comput. Chem., 16, 1164-1169.

57. Maier,J.A., Martinez,C., Kasavajhala,K., Wickstrom,L., Hauser,K.E. and Simmerling,C. (2015) ff14SB: Improving the Accuracy of Protein Side Chain and Backbone Parameters from ff99SB. J. Chem. Theory Comput., 11, 3696-3713.

58. Banáš,P., Hollas,D., Zgarbová,M., Jurečka,P., Orozco,M., Cheatham,T.E., Šponer,J. and Otyepka,M. (2010) Performance of molecular mechanics force fields for RNA simulations: Stability of UUCG and GNRA hairpins. J. Chem. Theory Comput., 6, 3836-3849.

59. Zgarbová,M., Otyepka,M., Šponer,J., Mládek,A., Banáš,P., Cheatham,T.E. and Jurečka,P. (2011) Refinement of the Cornell et al. Nucleic acids force field based on reference quantum chemical calculations of glycosidic torsion profiles. J. Chem. Theory Comput., 7, 2886-2902.

60. Götz,A.W., Williamson,M.J., Xu,D., Poole,D., Le Grand,S. and Walker,R.C. (2012) Routine microsecond molecular dynamics simulations with AMBER on GPUs. 1. generalized born. J. Chem. Theory Comput., 8, 1542-1555.

61. Salomon-Ferrer,R., Götz,A.W., Poole,D., Le Grand,S. and Walker,R.C. (2013) Routine microsecond molecular dynamics simulations with AMBER on GPUs. 2. Explicit solvent particle mesh ewald. J. Chem. Theory Comput., 9, 3878-3888.

62. Cirera,S. and Busk,P.K. (2014) Quantification of miRNAs by a simple and specific qPCR method. Methods Mol. Biol., 1182, 73-81.

63. Dietrich,L., Rathmer,B., Ewan,K., Bange,T., Heinrichs,S., Dale,T.C., Schade,D. and Grossmann,T.N. (2017) Cell Permeable Stapled Peptide Inhibitor of Wnt Signaling that Targets $\beta$-Catenin Protein-Protein Interactions. Cell Chem. Biol., 24, 958-968.e5.

64. Liu,S.R., Zhou,J.J., Hu,C.G., Wei,C.L. and Zhang,J.Z. (2017) MicroRNA-mediated gene silencing in plant defense and viral counter-defense. Front. Microbiol., 8, 1-12.

65. Bartel,D.P. (2009) MicroRNAs: Target Recognition and Regulatory Functions. Cell, 136, 215-233.

66. Lin,S. and Gregory,R.I. (2015) MicroRNA biogenesis pathways in cancer. Nat. Rev. Cancer, 15, 321-333.

67. Krichevsky,A.M. and Gabriely,G. (2009) miR-21: A small multi-faceted RNA. J. Cell. Mol. Med., 13, 39-53.

68. McLoughlin,N.M., Mueller,C. and Grossmann,T.N. (2018) The Therapeutic Potential of PTEN Modulation: Targeting Strategies from Gene to Protein. Cell Chem. Biol., 25, 19-29.

69. Wilson,W.D., Tanious,F.A., Fernandez-Saiz,M. and Rigl,C.T. (1997) Evaluation of drug-nucleic acid interactions by thermal melting curves. Methods Mol. Biol., 90, 219-240. 
70. Quemener,A.M., Bachelot,L., Forestier,A., Donnou-Fournet,E., Gilot,D. and Galibert,M. (2020) The powerful world of antisense oligonucleotides: From bench to bedside. Wiley Interdiscip. Rev. RNA, 11, e1594.

71. Childs-Disney,J.L., Hoskins,J., Rzuczek,S.G., Thornton,C.A. and Disney,M.D. (2012) Rationally designed small molecules targeting the RNA that causes myotonic dystrophy type 1 are potently bioactive. ACS Chem. Biol., 7, 856-862.

72. Gareiss,P.C., Sobczak,K., McNaughton,B.R., Palde,P.B., Thornton,C.A. and Miller,B.L. (2008) Dynamic combinatorial selection of molecules capable of inhibiting the (CUG) repeat RNAMBNL1 interaction in vitro: Discovery of lead compounds targeting myotonic dystrophy (DM1). J. Am. Chem. Soc., 130, 16254-16261.

73. Horne,W.S. and Grossmann,T.N. (2020) Proteomimetics as protein-inspired scaffolds with defined tertiary folding patterns. Nat. Chem., 12, 331-337.

74. Boga,S., Bouzada,D., García Peña,D., Vázquez López,M. and Vázquez,M.E. (2018) SequenceSpecific DNA Recognition with Designed Peptides. European J. Org. Chem., 2018, 249-261.

75. Lombardo,C.M., Kumar,V., Douat,C., Rosu,F., Mergny,J.L., Salgado,G.F. and Guichard,G. (2019) Design and Structure Determination of a Composite Zinc Finger Containing a Nonpeptide Foldamer Helical Domain. J. Am. Chem. Soc., 141, 2516-2525.

76. Hyun,S.L. and Schultz,P.G. (2008) Biosynthesis of a site-specific DNA cleaving protein. J. Am. Chem. Soc., 130, 13194-13195.

77. Learte-Aymamí,S., Rodríguez,J., Vázquez,M.E. and Mascareñas,J.L. (2020) Assembly of a Ternary Metallopeptide Complex at Specific DNA Sites Mediated by an AT- Hook Adaptor. Chem. - A Eur. J., 26, 8875-8878.

78. Neidle,S. (1999) Oxford Handbook of Nucleic Acid Structure 1st ed. Oxford University Press, Oxford, UK.

79. Chen,Y. and Varani,G. (2013) Engineering RNA-binding proteins for biology. FEBS J., 280, 37343754.

80. Disney,M.D., Winkelsas,A.M., Velagapudi,S.P., Southern,M., Fallahi,M. and Childs-Disney,J.L. (2016) Inforna 2.0: A Platform for the Sequence-Based Design of Small Molecules Targeting Structured RNAs. ACS Chem. Biol., 11, 1720-1728. 